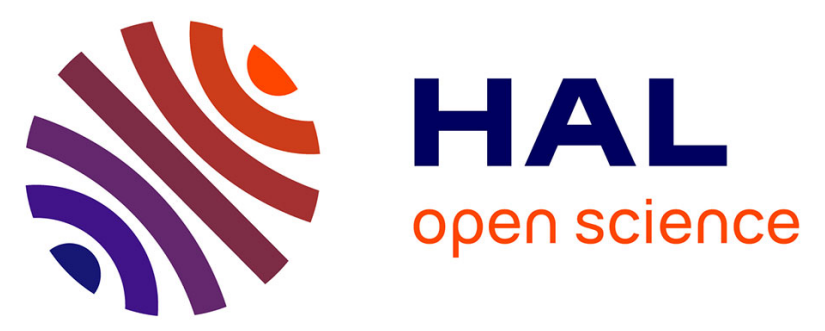

\title{
Channel Modelling and Performance Limits of Vehicular Visible Light Communication Systems
}

\author{
Mehdi Karbalayghareh, Farshad Miramirkhani, Hossien B Eldeeb, Refik \\ Caglar Kizilirmak, Sadiq M Sait, Murat Uysal
}

\section{- To cite this version:}

Mehdi Karbalayghareh, Farshad Miramirkhani, Hossien B Eldeeb, Refik Caglar Kizilirmak, Sadiq M Sait, et al.. Channel Modelling and Performance Limits of Vehicular Visible Light Communication Systems. IEEE Transactions on Vehicular Technology, 2020, 69 (7), pp.6891-6901. 10.1109/TVT.2020.2993294 . hal-03341267

\section{HAL Id: hal-03341267 \\ https://hal-amu.archives-ouvertes.fr/hal-03341267}

Submitted on 10 Sep 2021

HAL is a multi-disciplinary open access archive for the deposit and dissemination of scientific research documents, whether they are published or not. The documents may come from teaching and research institutions in France or abroad, or from public or private research centers.
L'archive ouverte pluridisciplinaire HAL, est destinée au dépôt et à la diffusion de documents scientifiques de niveau recherche, publiés ou non, émanant des établissements d'enseignement et de recherche français ou étrangers, des laboratoires publics ou privés. 


\title{
Channel Modelling and Performance Limits of Vehicular Visible Light Communication Systems
}

\author{
Mehdi Karbalayghareh, Student Member, IEEE, Farshad Miramirkhani, Member, IEEE, \\ Hossien B. Eldeeb, Student Member, IEEE, Refik Caglar Kizilirmak, Member, IEEE, \\ Sadiq M. Sait, Senior Member, IEEE, and Murat Uysal, Fellow, IEEE
}

\begin{abstract}
Visible light communication (VLC) has been proposed as an alternative or complementary technology to radio frequency vehicular communications. Front and back vehicle lights can serve as wireless transmitters making VLC a natural vehicular connectivity solution. In this paper, we evaluate the performance limits of vehicular VLC systems. First, we use non-sequential ray tracing to obtain the channel impulse responses (CIRs) for vehicle-to-vehicle (V2V) link in various weather conditions. Based on these CIRs, we present a closedform path loss expression which builds upon the summation of geometrical loss and attenuation loss and takes into account asymmetrical patterns of vehicle light sources and geometry of V2V transmission. The proposed expression is an explicit function of link distance, lateral shift between two vehicles, weather type (quantified by the extinction coefficient), transmitter beam divergence angle and receiver aperture diameter. Then, we utilize this expression to determine the maximum achievable link distance of $\mathrm{V} 2 \mathrm{~V}$ systems for clear, rainy and foggy weather conditions while ensuring a targeted bit error rate.
\end{abstract}

Index Terms-Visible light communications (VLC), vehicular communications, ray tracing, single photon avalanche diode (SPAD).

\section{INTRODUCTION}

$\mathbf{I}$ NTELLIGENT Transportation Systems (ITSs) are built upon cooperation, connectivity, and automation of vehicles, and are expected to improve the safety, efficiency, and sustainability of passengers and freight while enhancing the comfort of driving [1]. The practical implementation of ITSs require highly reliable, robust and scalable vehicle-to-

Copyright (c) 2015 IEEE. Personal use of this material is permitted. However, permission to use this material for any other purposes must be obtained from the IEEE by sending a request to pubs-permissions@ieee.org.

This paper was presented in part at IEEE Vehicular Technology Conference (VTC'18-Spring) and IEEE Wireless Communications and Networking Conference (WCNC'19). The work of H. B. Eldeeb was supported by the Horizon 2020 MSC ITN (VISION) under Grant 764461. Sadiq M. Sait would like to acknowledge KFUPM for support. The work of M. Uysal was supported by the Turkish Scientific and Research Council (TUBITAK) under Grant 215E311.

M. Karbalayghareh is with The University of Texas at Dallas, Department of Electrical and Computer Engineering, 75080 Richardson, TX, USA (e-mail: mehdi.karbalayghareh@UTDallas.edu).

F. Miramirkhani is with Isik University, Department of Electrical and Electronics Engineering, 34980 Istanbul, Turkey (e-mail: farshad.miramirkhani@isikun.edu.tr).

H. B. Eldeeb, and M. Uysal are with Ozyegin University, Department of Electrical and Electronics Engineering, 34794 Istanbul, Turkey (e-mails: hossien.eldeeb@ozu.edu.tr, and murat.uysal@ozyegin.edu.tr).

R. C. Kizilirmak is with Nazarbayev University, Department of Electrical and Computer Engineering, 010000 Astana, Kazakhstan (e-mail: refik.kizilirmak@nu.edu.kz).

Sadiq M. Sait is with King Fahd University of Petroleum \& Minerals, Computer Engineering Department, and, Center for Communications and IT Research, 31261 Dhahran, Saudi Arabia (e-mail: sadiq@kfupm.edu.sa). everything (V2X) communication solutions enabling vehicleto-infrastructure (V2I) and vehicle-to-vehicle (V2V) connectivity. Research efforts and standardization activities on V2X have been so far centered around radio frequency (RF) technologies [2-4]. In 2010, as an amendment of the IEEE 802.11 WiFi standard, IEEE $802.11 \mathrm{p}$ standard was introduced to support V2X communications in the $5.9 \mathrm{GHz}$ band allocated for ITS applications [1]. In 2017, cellular-based V2V connectivity solution, known as LTE-V, was introduced as a part of 3GPP Release 14 [5].

Since the current market penetration of V2X solutions is relatively low, the allocated RF bands are considered sufficient at the time being. However, in the near future, high interference levels can be experienced in limited RF bands particularly in high-density traffic scenarios. Channel congestion will result in longer delays and degrade the packet rate. To address such issues, visible light communications (VLC) has been proposed as an alternative vehicular access solution to RF-based V2X communications [6-8]. VLC takes advantage of widely available light emitting diodes (LEDs) and utilizes them as wireless transmitters in addition to their primary illumination function. Since automotive manufacturers are increasingly using LED-based exterior lighting, front and back vehicle lights can serve as wireless transmitters making VLC a natural vehicular connectivity solution. In addition to interference immunity, VLC has the potential to achieve very high data rates on the order of Gbps with off-the-shelf LEDs [9] or even more with custom design LEDs [10]. With such attractive features, vehicular VLC has received an increasing attention lately and several topics such as physical layer design, upper layer network protocols and integration with RF-based solutions for hybrid systems have been studied in the recent literature, see the survey in [11] and the references therein.

As in any other communication system, the propagation channel dictates the fundamental performance limits on vehicular VLC systems. Extensive studies of indoor VLC channel modelling have been already reported [12, 13]. However, operation in outdoor medium brings additional challenges and requires the development of dedicated vehicular VLC channels models. Initial works on vehicular VLC $[14,15]$ assume the line-of-sight (LOS) channel model. While this model is suitable for the indoor LED luminaires with Lambertian pattern, it fails to capture the illumination characteristics of automotive low-beam and high-beam headlamps with asymmetrical intensity distributions. To address such shortcomings, a piecewise 
Lambertian channel model was proposed in [16]. Intensity patterns for headlights were measured in experimental works [17-19] and used in the performance analysis of V2V VLC systems. The work in [20] further presented a closed-form path loss formula as a function of distance based on data fitting to measurement results. This formula is however limited to clear weather conditions. Furthermore, since it is obtained based on data fitting, the relation of system and channel parameters to path loss is not determined.

A critical factor that needs to be further taken into account in vehicular VLC channel modelling is the road surface. In [17], Lee et al. used Monte Carlo ray tracing and obtained vehicular VLC channel delay profiles for an asphalt road. In [18] and [19], Luo et al. developed a channel model for V2V transmissions up to $70 \mathrm{~m}$ over dry and wet asphalt. These works however assume fixed reflectance values for the vehicle coating (paint) and road surface. Such an assumption can be justified for infrared wavelengths; however, in the visible light band, reflectance is wavelength-dependent and this should be considered for a realistic channel modelling.

Another critical factor in the development of vehicular VLC channels is the impact of weather conditions. While there are earlier works on infrared channel modelling in the presence of different weather conditions [21], these are not applicable to vehicular VLC links due to different operation wavelength. In an effort to address this, the effect of artificially generated rain and fog on the received optical signal was quantified for a red coloured taillight LED using a laboratory chamber in [22] and [23]. In [24], a V2V link with a high-beam headlamp as the transmitter was considered and a path loss expression was developed for clear, rain and fog conditions. This is however valid only for short link ranges up to $20 \mathrm{~m}$. In addition to that, it is obtained via data fitting; therefore, the dependence of the expression on system parameters is not clear.

In the light of above literature survey, it is obvious that there is a need for the development of a comprehensive channel model simultaneously taking into account all inherent characteristics of vehicular VLC, i.e., asymmetrical radiation pattern of headlamps, weather effects, and effect from road reflections. In an effort to address this research gap, we present a closedform path loss expression for vehicular VLC as a function of system and channel parameters. Our proposed expression builds upon the summation of geometrical loss and attenuation loss and takes into account asymmetrical patterns of light sources and geometry of $\mathrm{V} 2 \mathrm{~V}$ transmission. It is an explicit function of link distance, lateral shift between two vehicles, weather type (quantified by the extinction coefficient), transmitter beam divergence angle and receiver aperture diameter. Based on extensive non-sequential ray tracing simulations, we obtain the channel impulse responses (CIRs) for a given vehicular scenario in various weather conditions, i.e., clear, rainy and foggy, and determine correction coefficients for each weather type. In the second part of the paper, we use this path loss expression to analyze the performance of a vehicular VLC system. To enable long range transmission, a single photon avalanche diode (SPAD) is employed as the receiver. We assume that the output photon number of SPAD receiver is modelled with Poisson statistics and determine the maximum achievable link distance to ensure a pre-defined value of bit error rate (BER).

The rest of the paper is organized as follows. In Section II, we develop the path loss expression for vehicular VLC and present numerical and simulation results to demonstrate the effect of weather conditions and system parameters on the path loss. In Section III, we investigate the maximum transmission range to satisfy a targeted BER value for the V2V VLC system under consideration in various weather conditions. In Section IV, we present system performance results and discussions. Finally, we provide concluding remarks in Section V.

\section{Vehicular VLC Path Los Model}

In this section, we first propose a closed-form path loss expression, then validate it through ray tracing simulations.

\section{A. Path Loss Expression}

We consider a single-lane road with a width of $w_{r}$. We assume that two vehicles follow each other in the same lane with a possible lateral shift of $d_{h}$. Each vehicle has a width of $w_{c}$ and they are separated from each other with a longitudinal distance of $d$. The two high-beam headlamps (denoted by TX1 and TX2) are adopted as wireless transmitters which are horizontally separated from each other with a distance of $d_{t x}$. A single photodetector (denoted by RX), placed at the center of the back of the other vehicle, acts as a wireless receiver. It has an aperture diameter of $D_{R}$ and a field of view angle of FOV. $L_{i}$ is the propagation distance from the $i$ th transmitter, $i=1,2$, to the receiver. The irradiance angle of the $i$ th transmitter is given by $\theta_{i}$ while the incident angle is defined by $\phi_{i}$.

The path loss consists of two main components, i.e, attenuation loss and geometrical loss. Attenuation loss is a result of scattering and absorption while the geometrical loss [25] is a result of the fact that the transmitted beam spreads to a size larger than the receive aperture. The geometrical loss between the $i$ th transmitter and the receiver can be expressed as

$$
P L_{g e o, i}=10 \log _{10}\left(\frac{D_{R}\left(\cos \left(\theta_{i}\right)\right)^{1 / \varepsilon}}{\zeta L_{i}}\right)^{2} i=1,2
$$

where we introduced two correction coefficients $(\zeta$ and $\varepsilon$ ) to take into account weather conditions and asymmetrical pattern of headlamp. The values of these correction coefficients are determined through ray tracing simulation study which will be discussed later.

The well-known Beer-Lambert formula defines the attenuation loss [26, Chapter 3] and builds upon the implicit assumption that scattered photons cannot be captured by the receiver. In order to take into factor that some of the scattered rays could be received by the photodetector after some reflections, this can be modified as

$$
P L_{a t t, i}=10 \log _{10}\left(\exp \left(-c L_{i}\left(\frac{D_{R}}{\zeta L_{i}}\right)^{\frac{\varepsilon}{2}}\right)\right)
$$

where $c$ stands for the extinction coefficient for a specific weather type and an additional term is introduced in the negative exponential that is proportional to the geometrical propagation of the light source [27]. Based on (1) and (2), 


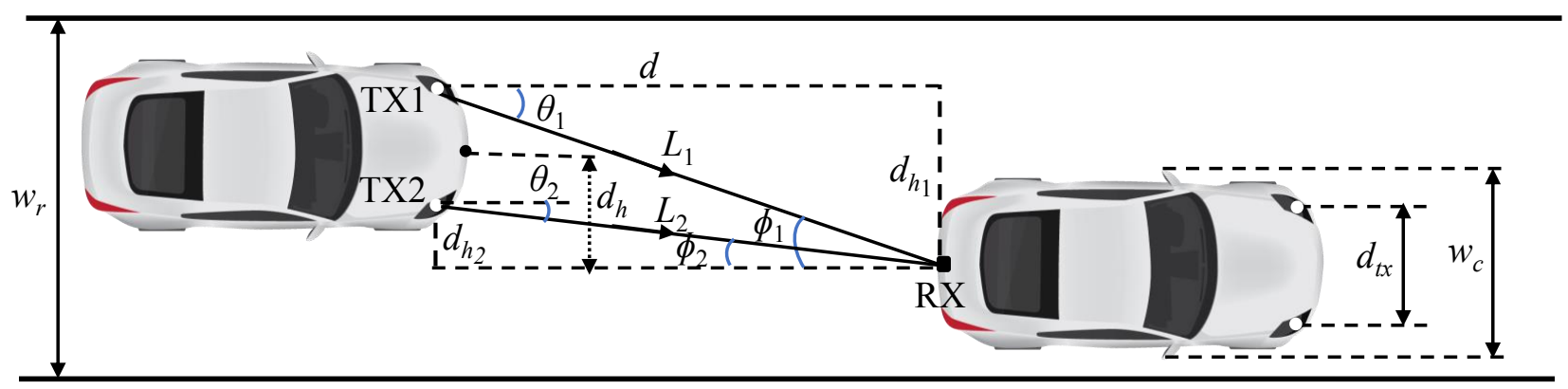

Fig. 1: Vehicle-to-vehicle scenario

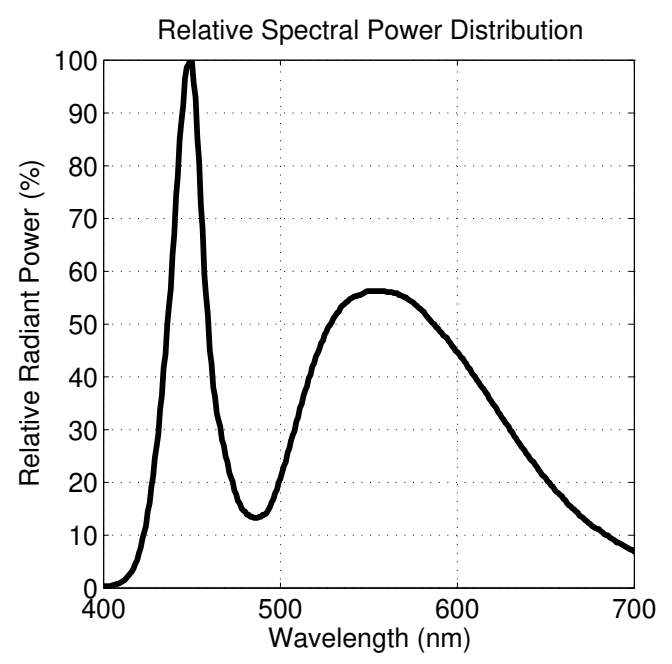

(a)

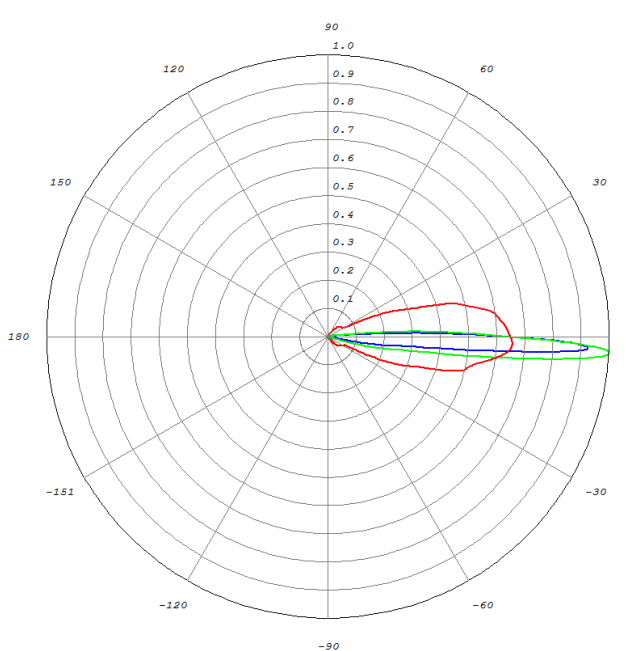

(b)

Fig. 2: (a) Relative spectral power distribution and (b) relative intensity distributions of high-beam headlamp

the path loss for the link between the $i$ th transmitter and the receiver can be expressed as

$$
P L_{i}=10 \log _{10}\left(\left(\frac{D_{R}\left(\cos \left(\theta_{i}\right)\right)^{1 / \varepsilon}}{\zeta L_{i}}\right)^{2} \exp \left(-c L_{i}\left(\frac{D_{R}}{\zeta L_{i}}\right)^{\frac{\varepsilon}{2}}\right)\right)
$$

From the geometry of layout in Fig. 1, it can be readily checked that $L_{i}=\sqrt{d^{2}+d_{h_{i}}^{2}}$ where $d_{h_{i}}=d_{h} \pm d_{t x} / 2$ and $\theta_{i}=\arccos \left(d / L_{i}\right)$. Noting the use of dual transmitters and replacing $L_{i}$ and $\theta_{i}$ within (3), we can obtain the overall path loss as (4).

\section{B. Channel Modeling Approach}

In the following, we conduct a simulation study in Zemax ${ }^{\circledR}$ to validate the proposed path loss expression and determine the values of correction coefficients for the vehicular channels under consideration. Our channel modelling approach builds on non-sequential ray tracing features of Zemax ${ }^{\circledR}$ which allows rays to propagate through the environment in any order and allows rays to be scattered and reflected back to an object that they have already encountered. This property makes nonsequential mode ideal for impulse response modelling. This method was originally adopted to model indoor VLC channels $[12,13]$ and also applied to the modelling of underwater VLC channels [27].

First, a 3D model of vehicular scenario (including vehicles, road etc) is constructed in Zemax ${ }^{\circledR}$. As inputs, we provide the specifications of vehicular light sources acting as transmitters (i.e., spectral power distribution and intensity distribution) and photodetectors placed on the vehicles for reception (i.e., location on the vehicle, aperture size and field of view). We further specify the optical characteristics of the vehicle coating and road surface by defining their wavelength-dependent reflectance values. To reflect the effects of weather conditions, we need to define the atmospheric propagation medium. Zemax ${ }^{\circledR}$ built-in function "Bulk Scatter" allows providing the refractive index, radius and density of particles as input parameters based on Mie model [26, Chapter

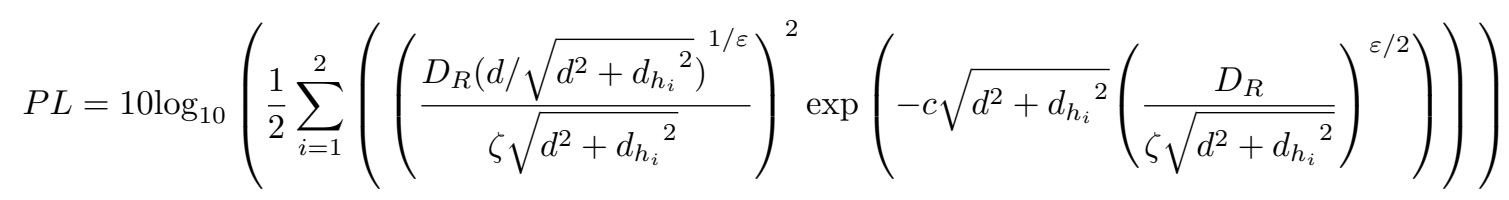


TABLE I: Characteristics of various weather types [26, Chapter 3]

\begin{tabular}{|c|c|c|c|c|}
\hline & Particle Index & Size $(\boldsymbol{\mu m})$ & Density $\left(\mathbf{c m}^{-3}\right)$ & Extinction Coefficient $\left(\mathbf{m}^{-1}\right)$ \\
\hline Clear Weather & 1.000277 & $10^{-4}$ & $10^{19}$ & $\approx 0$ \\
\hline Rain & 1.33 & 100 & 0.1 & $\approx 0$ \\
\hline Moderate Fog & 1.33 & 10 & 12.45 & 0.00782 \\
\hline Thick Fog & 1.33 & 10 & 24.91 & 0.01565 \\
\hline
\end{tabular}

3]. The associated values for various weather types can be found in Table I [26, Chapter 3].

After simulation environment is created, non-sequential ray tracing is run to determine the CIR. Zemax ${ }^{\circledR}$ generates an output file including the received power and path length from source to detector for each ray. These are then imported to Matlab ${ }^{\circledR}$ to construct the CIR. Assume that $N_{i}$ is the number of rays received at the detector from the $i$ th transmitter. Let $P_{i, j}$ and $\tau_{i, j}$ respectively denote the power and propagation time of the $j$ th ray, $j=1, \ldots, N_{i}$ received from the $i$ th transmitter. Then, the CIR can be constructed as $h_{\text {sim }}(t)=\sum_{i=1}^{2}\left(\sum_{j=1}^{N_{i}} P_{i, j} \delta\left(t-\tau_{i, j}\right)\right)$ where $\delta(t)$ is the Dirac delta function. The path loss can be calculated as [28]

$$
P L_{\text {sim }}=10 \log _{10}\left(\int_{0}^{\infty} h_{\text {sim }}(t) d t\right)
$$

\section{Simulation Study}

In our simulation study, we construct the 3D model of vehicular scenario in Fig. 1. CAD objects of vehicles are imported and processed in Zemax ${ }^{\circledR}$ "Part Designer" which is an interface that allows to create and manipulate user-defined $3 \mathrm{D}$ geometries. The surface coating for two vehicles is chosen as black gloss paint.

As the high-beam headlamp of the vehicle, a commercially available white light LED (Philips Luxeon Rebel) is used [29]. This headlamp is modelled in the simulation platform by defining the relative spectral power distribution of the LED (see Fig. 2.a) and the photometric data which contains the luminous intensity in all different planes. Specifically, the photometric data is imported as an .IES file to the software. As an example, different cross sections are shown in Fig. 2.b to demonstrate the asymmetrical intensity pattern of headlamp. Blue curve shows the intensity distribution of headlamp when one looks from side (e.g., from pavement) while the red one shows the same pattern when one looks downward from top. The green curve shows the pattern when one looks downward at a 45 degrees angle. These planes are obtained through "source viewer" feature of Zemax ${ }^{\circledR}$ which provides the directivity plots of the transmitter in different scan angles. In the simulation study, right and left headlamps with the above characteristics are placed in the front side of the first vehicle as the transmitters. A total unit (normalized) power is assumed in the simulations and the CIRs are then scaled for a given transmit power. A photodetector is placed at the back (bumper) of the other vehicle and serves as the receiver. The receiver has an aperture size of $D_{R}=1 \mathrm{~cm}$ and field of view (FOV) of $180^{\circ}$.

\footnotetext{
${ }^{1}$ It is possible to improve signal-to-noise ratio (SNR) by narrowing the receiver FOV through the use of a proper lens. However, a narrow FOV might be problematic for mobile conditions as encountered in vehicular VLC. Therefore, we prefer a large FOV to maximize reception angle.
}

TABLE II: Correction coefficients for different weather conditions

\begin{tabular}{|c|c|c|}
\hline & $\zeta(\mathrm{rad})$ & $\varepsilon$ \\
\hline Clear Weather & 0.1585 & 0.0175 \\
\hline Rain & 0.1598 & 0.0174 \\
\hline Moderate Fog & 0.1600 & 0.0172 \\
\hline Thick Fog & 0.1550 & 0.0170 \\
\hline
\end{tabular}

As road type, we consider categorizations defined by the International Commission on Illumination (CIE) [30] and assume R2 type road. This defines an asphalt road consisting of $60 \%$ gravel sized larger than $10 \mathrm{~mm}$. To simulate clear and foggy weathers, mixed specular and diffuse reflections are assumed while mostly specular reflections are considered for the wet road surface in rain [19].

In our simulation study, it is assumed that there is an initial separation of longitudinal distance meters between two cars. For a range of 100 meters, we obtain the CIRs through all points with 5 meters inter-distance. The simulations are conducted for clear weather, rain, moderate fog (i.e., visibility of $500 \mathrm{~m}$ ) [31] and thick fog (i.e., visibility of $250 \mathrm{~m}$ ) [31]. In the following, we present the proposed path loss expression versus distance for the weather conditions under consideration along with simulation results. Corresponding $\zeta$ and $\varepsilon$ values are summarized in Table II. In addition to simulations, we include the following three benchmarks.

1) Path loss calculated based on the assumption of Lambertian source and Beer-Lambert formula: As earlier discussed, some initial works on vehicular VLC consider Lambertian source model [14, 15] and Beer-lambert formula. Under these assumptions and noting that we have two light sources, we can write the path loss as (6) [26, Chapter 3], [32] where $m=-1 / \log _{2}\left(\cos \left(\Phi_{1 / 2}\right)\right)$ is the order of Lambertian emission with $\Phi_{1 / 2}$ denoting the semi-angle of the LED. In (6), the first term denotes geometrical loss under the assumption of Lambertian source and the second term is the attenuation loss based on Beer-Lambert formula.

2) Path loss calculated based on the assumption of asymmetrical source and Beer Lambert formula: Combining (1) and the second term of (6), we have (7).

3) Path loss formula introduced in [20] based on the curve fitting to the empirical data: This formula is given by

$$
P L=\alpha+10 \beta \log _{10}\left(\frac{1}{d+\gamma}\right)
$$

where the coefficients $\alpha, \beta$ and $\gamma$ are found via data fitting to measurement results. The experiment was conducted under clear weather conditions at night. In the experimental set-up, two vehicles follow each other in the same lane where low-beam headlamp is used as the transmitter and a receiver aperture area of is $9.8 \mathrm{~mm}$ $\times 9.8 \mathrm{~mm}$ (i.e., approximately $1 \mathrm{~cm}^{2}$ ) is assumed. 


$$
\begin{gathered}
P L=10 \log _{10}\left(\frac{1}{2} \sum_{i=1}^{2}\left(\left(\frac{\left(\pi D_{R}^{2} / 4\right)(m+1)}{2 \pi \sqrt{d^{2}+d_{h_{i}}^{2}}} \cos ^{m}\left(\theta_{i}\right) \cos \left(\phi_{i}\right)\right) \exp \left(-c \sqrt{d^{2}+d_{h i}^{2}}\right)\right)\right) \\
P L=10 \log _{10}\left(\frac{1}{2} \sum_{i=1}^{2}\left(\left(\frac{D_{R}\left(\cos \left(\theta_{i}\right)\right)^{1 / \varepsilon}}{\zeta L_{i}}\right)^{2} \exp \left(-c \sqrt{d^{2}+d_{h i}^{2}}\right)\right)\right)
\end{gathered}
$$

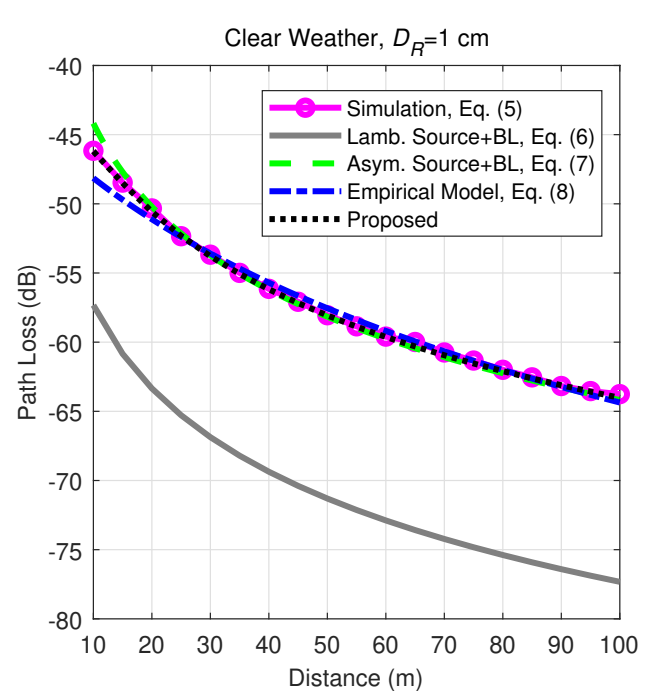

(a)

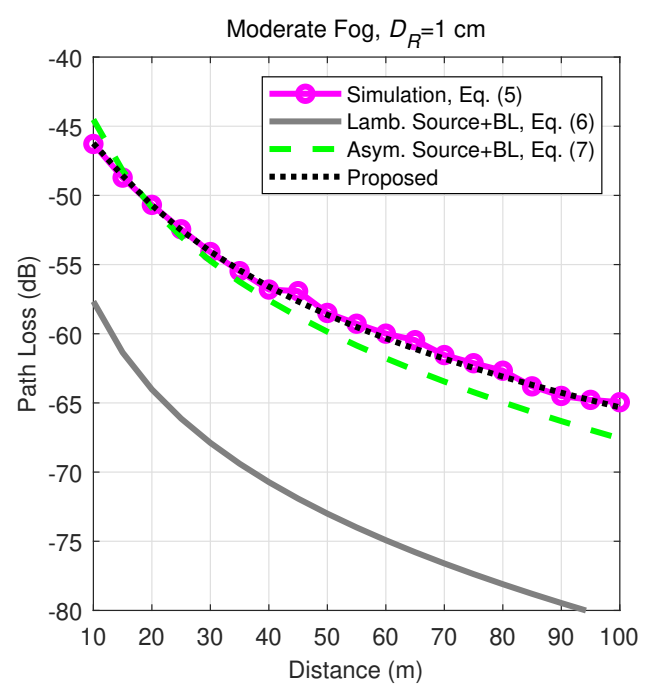

(c)

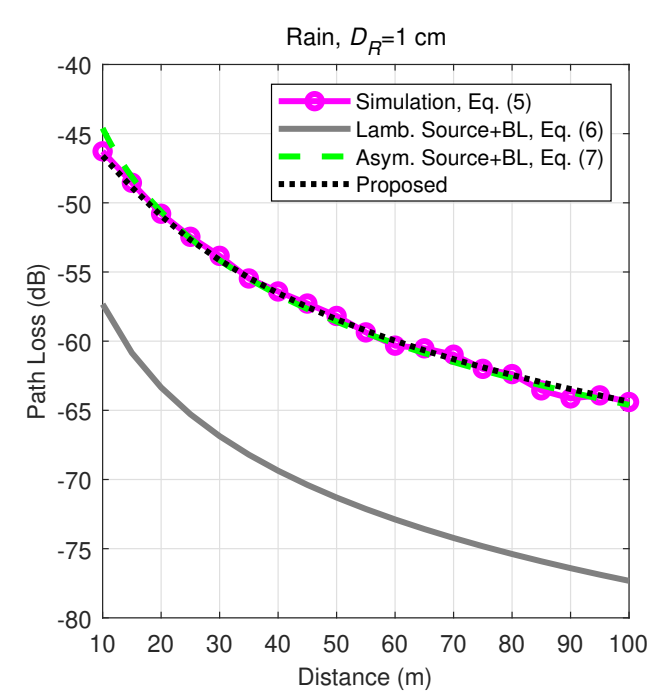

(b)

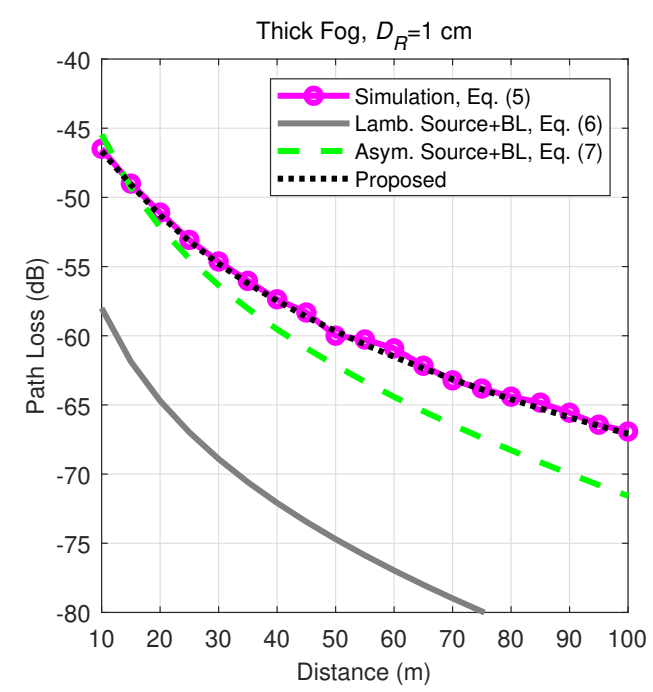

(d)

Fig. 3: Proposed path loss expression and comparison with simulation results and other benchmarks. Different weather conditions are assumed while the receiver aperture size is set as $D_{R}=1 \mathrm{~cm}$

The comparison of our results with two first benchmarks will be instrumental to have insight into the contribution of asymmetrical radiation pattern to the overall path loss. The third benchmark will let us to demonstrate the accuracy of simulations through comparison with experimental measurements.

In Fig. 3, we present our path loss model in (4) versus distance along with simulation results and the above three benchmarks. We assume receiver aperture diameter of $D_{R}=1 \mathrm{~cm}$. First of all, it can be verified from Fig. 3.a that our proposed path loss expression in (4) maintains a good fit with empirical model in (8) which was developed only for clear weather conditions. The deviation in small distances is due to this fact that the empirical model was obtained for lowbeam headlamp while our simulation study is based on highbeam headlamp. It should be noted that the intensity pattern of low-beam headlamp is more directed towards the road surface compared to the high-beam headlamp and hence the path loss for small distances is more pronounced in the empirical model.

Fig. 3 further reveals out that the proposed expression in 


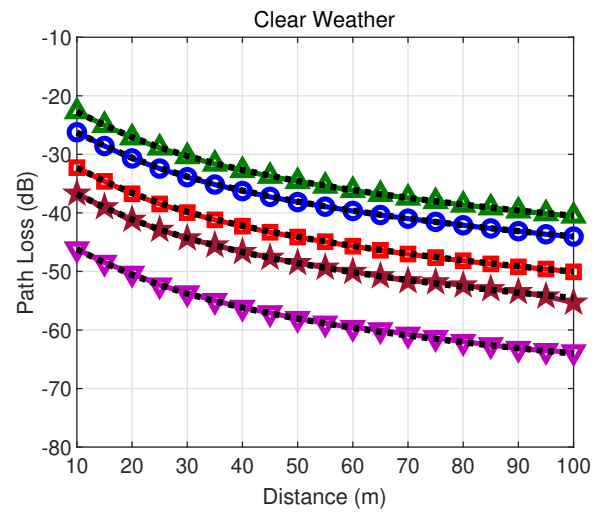

(a)

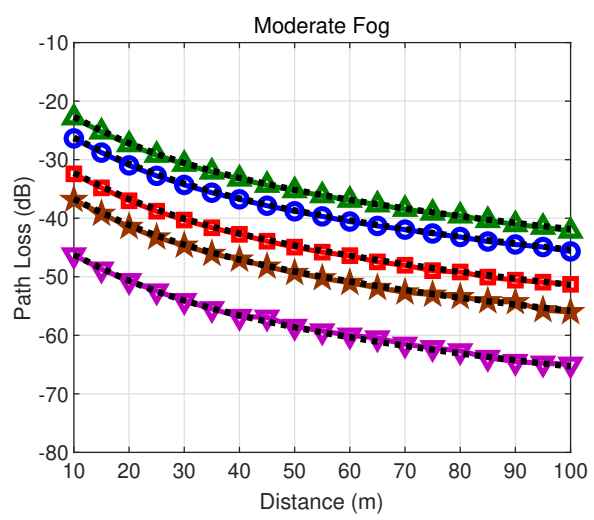

(c)
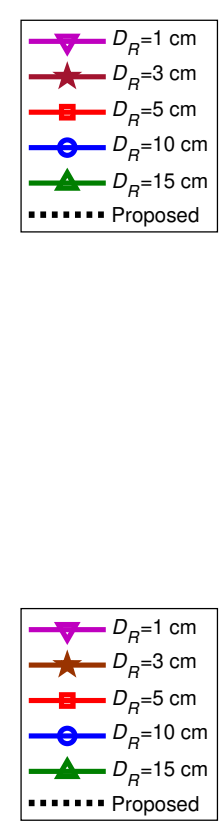
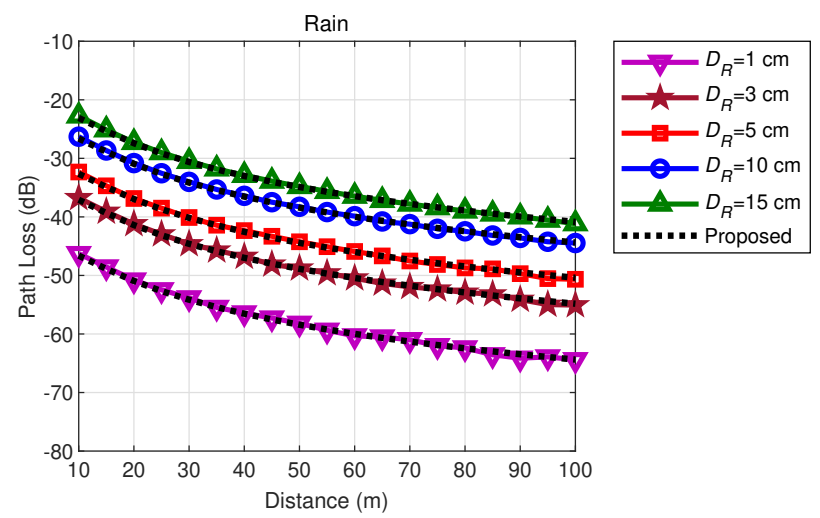

(b)
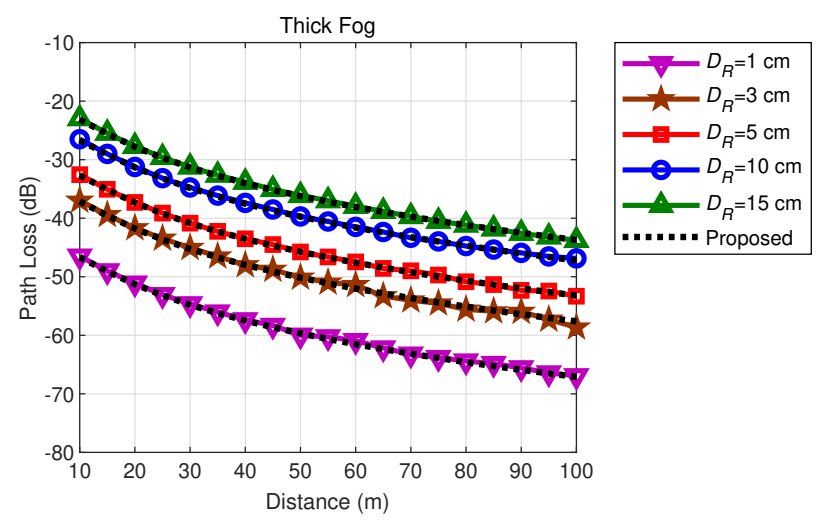

(d)

Fig. 4: Proposed path loss expression for different receiver aperture diameters under different weather conditions

(4) has a "good fit" 2 with simulation results for all weather conditions. In contrary, the benchmarking plots based on (6) and (7) exhibit some deviations. Particularly, the path loss obtained by Lambertian source is a significant overestimation over the actual path loss compared to an asymmetrical headlight, i.e., see the plot based on (6). It is further observed that Beer-Lambert formula is a good fit with simulation results only for clear and rainy weather conditions and deviates for foggy conditions, i.e., see the plot based on (7). Since the extinction coefficients of clear and rainy weathers are very small (i.e., $c \approx 0 \mathrm{~m}^{-1}$ ), the scattered rays can be ignored making Beer-Lambert formula valid for this case. In foggy weather conditions, the effect of scattering is significant. In this case, Beer-Lambert formula has a significant deviation from simulations. This deviation increases when the visibility decreases.

In Fig. 4, we investigate the validity of our proposed expression in (4) by varying the receiver aperture diameter. We assume $D_{R}=1 \mathrm{~cm}, 3 \mathrm{~cm}, 5 \mathrm{~cm}, 10 \mathrm{~cm}$ and $15 \mathrm{~cm}$. It can be verified that the path loss expression maintains a good fit for different values of receiver aperture diameters under various weather conditions. In Fig. 5, we consider different lateral shifts of $d_{h}=1 \mathrm{~m}$ and $1.5 \mathrm{~m}$ assuming clear weather conditions and $D_{R}=5 \mathrm{~cm}$. It is observed that the lateral shift brings negligible loss with respect to perfect alignment case of $d_{h}=0 \mathrm{~m}$.

\footnotetext{
${ }^{2}$ As a measure of data fitting accuracy, we use "R-squared $\left(R^{2}\right)$ " [33] which is said to be a "good fit" when $R^{2}$ exceeds 0.95 [34].
}

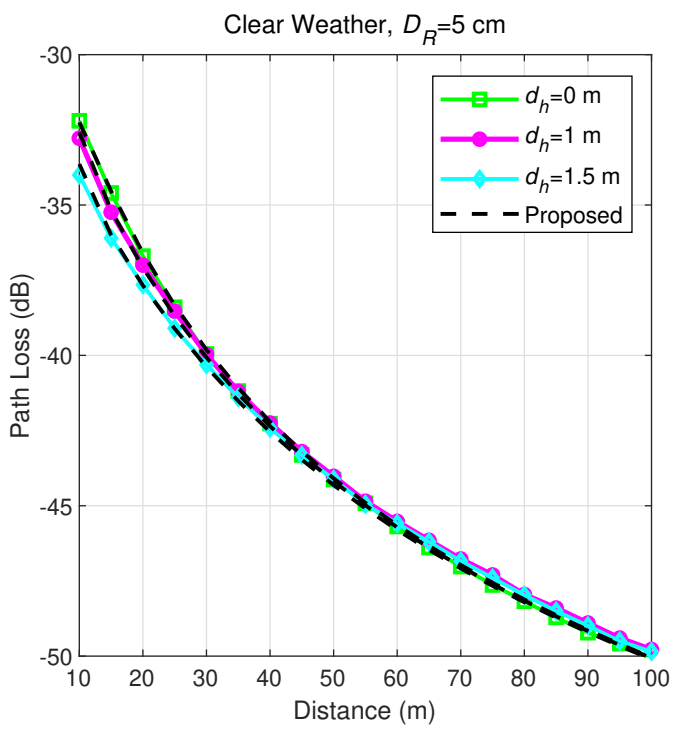

Fig. 5: Comparison of proposed path loss expression with simulation results for different lateral shifts

\section{Maximum Achievable Distance}

In this section, we investigate the error rate performance of vehicular VLC links and determine the achievable transmission distances in various weather conditions. We consider the use of SPAD which is known with their superior performance to detect weak signals $[35,36]$. SPADs were considered as VLC receivers in the literature for harsh propagation envi- 
ronments such as underwater [37-39] and gas pipelines [40]. However, their use was not investigated before in the context of vehicular VLC systems.

Most practical VLC systems use intensity modulation and direct detection. As such, we assume that unipolar on-off keying (OOK) is employed with an average optical power of $P_{0}$ and bit time duration of $T_{b}$. The power is set as $P_{t}=0$ and $P_{t}=2 P_{0}$, respectively for transmitting zeros and ones. Let $N_{S P A D}$ and $C_{F F}$ denote respectively the size and the fill factor (FF) of the SPAD array. The number of received photons is given by [41]

$$
z=N_{S P A D}\left(C_{F F} \eta P_{t} h+C_{F F} N_{b}+N_{D C R}\right) T_{b}
$$

where $h$ is the channel coefficient, $N_{D C R}$ is the dark count ratio, $N_{b}$ is the background light noise ratio and $\eta=C_{P D E} \lambda / h_{\text {Planck }} v$. Here, $C_{P D E}$ is the photon detection efficiency, $\lambda$ is the wavelength, $h_{\text {Planck }}$ is the Planck's constant and $v$ is the light speed. Since we are interested in finding the maximum achievable distance, we can impose the assumption of $d \gg d_{h_{i}}$. Therefore, (4) can be simplified as

$$
P L=10 \log _{10}(\underbrace{\left(\frac{D_{R}}{\zeta d}\right)^{2} \exp \left(-c d\left(\frac{D_{R}}{\zeta d}\right)^{\varepsilon / 2}\right)}_{h})
$$

Poisson distribution is widely used to statistically model the SPAD receiver. The probability mass function of the output photon numbers is given by $\operatorname{Pr}(z)=\mu^{z} e^{-\mu} / z$ ! where $\mu=E(z)$ denotes the mean. The corresponding received photon numbers for the transmission of zeros and ones are then calculated as $\mu_{0}=N_{S P A D}\left(C_{F F} N_{b}+N_{D C R}\right) T_{b}$ and $\mu_{1}=N_{S P A D} C_{F F} \eta P_{t} h T_{b}+\mu_{0}$, respectively. For equally likely message signals, it can be shown that the optimum detection threshold is $z_{\mathrm{th}}=\left(\mu_{1}-\mu_{0}\right) / \ln \left(\mu_{1} / \mu_{0}\right)$. BER for OOK under Poisson distribution can be then written as [41, 42]

$$
P_{e}=\frac{1}{2} \sum_{z=z_{\mathrm{th}}+1}^{\infty} \frac{\mu_{0}^{z}}{z !} e^{-\mu_{0}}+\frac{1}{2} \sum_{z=0}^{z_{\mathrm{th}}} \frac{\mu_{1}^{z}}{z !} e^{-\mu_{1}} .
$$

Using Gaussian approximation [43], the BER expression can be expressed as

$$
P_{e} \approx Q\left(\frac{\mu_{1}-\mu_{0}}{\sqrt{\mu_{1}}+\sqrt{\mu_{0}}}\right) .
$$

In the following, we calculate the maximum achievable distance to satisfy a targeted BER. First, we need to solve $h$ in (10) for $d$. After some mathematical manipulations on $h$, we obtain

$$
\begin{gathered}
\left.\left(\frac{c}{4}(2-\varepsilon)\left(\frac{D_{R}}{\zeta}\right)^{\varepsilon / 2} d^{\left(\frac{2-\varepsilon}{2}\right)}\right) e^{\left(\frac{c}{4}(2-\varepsilon)\left(\frac{D_{R}}{\zeta}\right)^{\varepsilon / 2} d\left(\frac{2-\varepsilon}{2}\right)\right.}\right) \\
=\frac{c}{4}(2-\varepsilon)\left(\frac{D_{R}}{\zeta}\right)^{\varepsilon / 2}\left(\frac{h \zeta^{2}}{D_{R}^{2}}\right)^{\left(\frac{\varepsilon-2}{4}\right)}
\end{gathered}
$$

Noticing that the left hand side (LHS) of (13) is in the form of $x e^{x}$, we can find its inverse by the Lambert-W function [44].
Let $\mathrm{W}\left(x e^{x}\right)=x$ denote the Lambert-W function. From (13), we obtain $d$ as

$$
d=\left(\frac{\mathrm{W}\left(\frac{c}{4}(2-\varepsilon)\left(\frac{D_{R}}{\zeta}\right) h^{\left(\frac{\varepsilon-2}{4}\right)}\right)}{\frac{c}{4}(2-\varepsilon)\left(\frac{D_{R}}{\zeta}\right)^{\varepsilon / 2}}\right)^{\frac{2}{2-\varepsilon}}
$$

Now, assume that $P_{e}^{\prime}$ denotes the value of targeted BER. To solve (12) for $h$ in (10), we first solve (12) for $\mu_{1}$. By taking inverse $Q$-function from both sides of (12), we have

$$
Q^{-1}\left(P_{e}^{\prime}\right) \approx \frac{\mu_{1}-\mu_{0}}{\sqrt{\mu_{1}}+\sqrt{\mu_{0}}} .
$$

By re-arranging (15), we have

$$
\mu_{1}-Q^{-1}\left(P_{e}^{\prime}\right) \sqrt{\mu_{1}}-\left(Q^{-1}\left(P_{e}^{\prime}\right) \sqrt{\mu_{0}}+\mu_{0}\right) \approx 0 .
$$

Then, $\mu_{1}$ can be obtained as

$\mu_{1} \approx\left(\frac{Q^{-1}\left(P_{e}^{\prime}\right)+\sqrt{\left(Q^{-1}\left(P_{e}^{\prime}\right)\right)^{2}+4\left(Q^{-1}\left(P_{e}^{\prime}\right) \sqrt{\mu_{0}}+\mu_{0}\right)}}{2}\right)^{2}$.

Based on (17) and noting that $\mu_{1}=N_{S P A D} C_{F F} \eta P_{t} h T_{b}+\mu_{0}$, we have (18). By rearranging (18), $h$ can be obtained as (19). By replacing (19) in (14), the maximum achievable link distance to achieve $P_{e}{ }^{\prime}$ can be obtained as (20).

\section{Performance Results and Discussions}

In this section, we present performance results of the $\mathrm{V} 2 \mathrm{~V}$ VLC system under different weather conditions in terms of achievable distance and also investigate the effect of system parameters on the performance. Unless otherwise stated, we assume average optical power of $P_{0}=-50 \mathrm{dBm}$ [35], receiver aperture diameter of $D_{R}=5 \mathrm{~cm}$, fill factor of $C_{F F}=0.5$ [45], array size of $N_{S P A D}=8 \times 8$ [43] and $N_{D C R}=7.27 \mathrm{kHz}$ [45]. The simulation parameters are summarized in Table III. Since white LED headlamps are used in vehicular applications, $\eta$ in (9) is calculated based on an average over the wide spectrum of visible band (400 nm-700 nm).

In Fig. 6, we illustrate the maximum achievable distances assuming clear weather, rain and fog for the above system configuration parameters. It is observed that a transmission range of $34.15 \mathrm{~m}$ is possible for clear weather at $P_{e}^{\prime}=10^{-6}$. This slightly reduces to $33.08 \mathrm{~m}$ in rain. On the other hand, for foggy condition, the achievable distances are $32.12 \mathrm{~m}$ and $30.01 \mathrm{~m}$ for moderate fog and thick fog, respectively.

In Fig. 7, we investigate the effect of receiver aperture size on the maximum achievable $\mathrm{V} 2 \mathrm{~V}$ distance under the assumption of thick fog. As observed from this figure, the transmission range increases with the increase in receiver aperture diameter. This is expected because a receiver with larger aperture is able to collect more energy. Mathematically speaking, the maximum achievable distance at $P_{e}{ }^{\prime}=10^{-6}$ is $6.39 \mathrm{~m}$ for $D_{R}=1 \mathrm{~cm}$. This increases to $18.4 \mathrm{~m}, 30.01$ $\mathrm{m}, 55.87 \mathrm{~m}$ and $77.64 \mathrm{~m}$ for $D_{R}=3 \mathrm{~cm}, D_{R}=5 \mathrm{~cm}, D_{R}$ $=10 \mathrm{~cm}$ and $D_{R}=15 \mathrm{~cm}$, respectively. According to EU Member States regulations, a safe trailing distance of $28 \mathrm{~m}$ 


$$
\begin{gathered}
N_{S P A D} C_{F F} \eta P_{t} h T_{b}+\mu_{0} \approx\left(\frac{Q^{-1}\left(P_{e}^{\prime}\right)+\sqrt{\left(Q^{-1}\left(P_{e}^{\prime}\right)\right)^{2}+4\left(Q^{-1}\left(P_{e}^{\prime}\right) \sqrt{\mu_{0}}+\mu_{0}\right)}}{2}\right)^{2} . \\
h \approx \frac{1}{N_{S P A D} C_{F F} \eta P_{t} T_{b}}\left(\frac{1}{4}\left(Q^{-1}\left(P_{e}^{\prime}\right)+\sqrt{\left(Q^{-1}\left(P_{e}^{\prime}\right)\right)^{2}+4\left(Q^{-1}\left(P_{e}^{\prime}\right) \sqrt{\mu_{0}}+\mu_{0}\right)}\right)^{2}-\mu_{0}\right) \\
d_{\max } \approx\left(\frac{\mathrm{W}\left(\frac{c}{4}(2-\varepsilon)\left(\frac{D_{R}}{\zeta}\right)\left(\frac{1}{N_{S P A D} C_{F F} \eta P_{t} T_{b}}\left(\frac{1}{4}\left(Q^{-1}\left(P_{e}^{\prime}\right)+\sqrt{\left(Q^{-1}\left(P_{e}^{\prime}\right)\right)^{2}+4\left(Q^{-1}\left(P_{e}^{\prime}\right) \sqrt{\mu_{0}}+\mu_{0}\right)}\right)^{2}-\mu_{0}\right)\right)^{\left(\frac{\varepsilon-2}{4}\right)}\right.}{\frac{c}{4}(2-\varepsilon)\left(\frac{D_{R}}{\zeta}\right)^{\varepsilon / 2}}\right)^{\frac{2}{2-\varepsilon}}
\end{gathered}
$$

TABLE III: Simulation parameters [45]

\begin{tabular}{|l|l|}
\hline \multicolumn{1}{|c|}{ Parameters } & \multicolumn{1}{c|}{ Values } \\
\hline Wavelength of light $(\lambda)$ & $400 \mathrm{~nm}-700 \mathrm{~nm}$ \\
\hline Speed of light $(\nu)$ & $3 \times 10^{8} \mathrm{~m} / \mathrm{s}$ \\
\hline Bit time duration $\left(T_{b}\right)$ & $1 \mu \mathrm{s}$ \\
\hline The PDE of the SPAD $\left(C_{P D E}\right)$ & $20 \%$ \\
\hline The DCR of the SPAD $\left(N_{D C R}\right)$ & $7.27 \mathrm{kHz}$ \\
\hline The FF of the SPAD $\left(C_{F F}\right)$ & 0.5 \\
\hline Size of the SPAD $\left(N_{S P A D}\right)$ & $8 \times 8$ \\
\hline
\end{tabular}

should be maintained between two vehicles based on 2-second rule assuming a travelling speed of $50 \mathrm{~km} / \mathrm{h} \mathrm{[46].} \mathrm{Therefore,}$ the aperture size of $D_{R}=5 \mathrm{~cm}$ is selected in the following.

In Fig. 8, we investigate the effect of background light noise ratio on the maximum achievable V2V distance. Here, we consider thick fog and vary background light noise ratio from much smaller than dark counts (i.e., at night with $N_{b}=0 \mathrm{~Hz}$ ) to much bigger than dark counts during daylight (i.e., $N_{b}=$ $100 \mathrm{kHz}$ ). It is observed that as background light noise ratio increases, the maximum achievable distance decreases. For example, under the assumption of $N_{b}=0 \mathrm{~Hz}$ and $P_{e}{ }^{\prime}=10^{-6}$, a $\mathrm{V} 2 \mathrm{~V}$ distance of $30.01 \mathrm{~m}$ is achievable. This reduces to $28.82 \mathrm{~m}$ and $25.04 \mathrm{~m}$ for $N_{b}=10 \mathrm{kHz}$ and $N_{b}=100 \mathrm{kHz}$, respectively.

In Fig. 9, we investigate the effect of SPAD parameters, i.e., fill factor and array size, on the maximum achievable V2V distance under the assumption of thick fog. It is observed that as fill factor and array size increase, the transmission range increases. For $N_{S P A D}=8 \times 8$ and $P_{e}{ }^{\prime}=10^{-6}$, a distance of $30.01 \mathrm{~m}$ is achievable assuming a fill factor of $C_{F F}=0.5$. This increases to $33.25 \mathrm{~m}$ and $39.86 \mathrm{~m}$ for $C_{F F}=0.64$ [43] and $C_{F F}=1$ (i.e., hypothetical case where the total array area is assumed to be active), respectively.

\section{Conclusions}

In this paper, we characterized the path loss of V2V VLC system as a function of weather type and system parameters. The proposed path loss expression takes into account the possibility of receiving scattered photons and asymmetrical intensity distribution of headlamps. We further used this path loss model to derive a closed-form expression for the maximum achievable $\mathrm{V} 2 \mathrm{~V}$ distance. We considered the deployment of SPADs as receivers where the output photon number is modeled with Poisson statistics. We derived the maximum

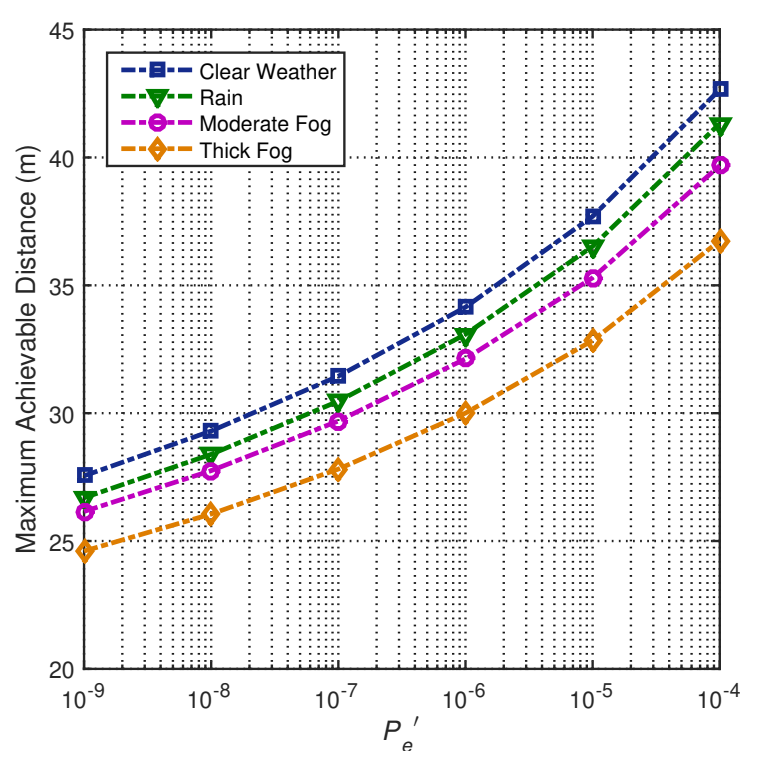

Fig. 6: Maximum achievable distance under different weather conditions

achievable V2V distance to satisfy a targeted BER value. Our results demonstrated that, with proper selection of system parameters, VLC can provide a reliable connectivity solution for V2V links.

\section{REFERENCES}

[1] A. Perallos, U. Hernandez-Jayo, I. J. G. Zuazola, and E. Onieva, Intelligent Transport Systems: Technologies and Applications, John Wiley \& Sons, 2015 .

[2] O. Karagiannis, O. Altintas, E. Ekici, G. Heijenk, B. Jarupan, K. Lin, and T. Weil, "Vehicular networking: A survey and tutorial on requirements, architectures, challenges, standards and solutions", IEEE Commun. Surveys Tut., vol. 13, no. 4, pp. 584-616, 2011.

[3] G. Araniti, C. Campolo, M. Condoluci, A. Iera, and A. Molinaro, "LTE for vehicular networking: A survey", ”, IEEE Commun. Mag., vol. 51, no. 5, pp. 148-157, 2013.

[4] K. Zheng, Q. Zheng, P. Chatzimisios, W. Xiang, and Y. Zhou, "Heterogeneous vehicular networking: a survey on architecture, challenges, and solutions", IEEE Commun. Surveys Tut., vol. 17, no. 4, pp. 2377-2396, 2015.

[5] R. Molina-Masegosa, and J. Gozalvez, "LTE-V for sidelink 5G V2X vehicular communications: A new 5G technology for short-range vehicleto-everything communications", IEEE Veh. Technol. Mag., vol. 12, no. 4, pp. 30-39, Dec. 2017.

[6] T. Yamazato, I. Takai, H. Okada, T. Fujii, T. Yendo, S. Arai, M. Andoh, T. Harada, K. Yasutomi, K. Kagawa, and S. Kawahito, "Image-sensor-based visible light communication for automotive applications", IEEE Commun. Mag., vol. 52, no. 7, pp. 88-97, 2014. 


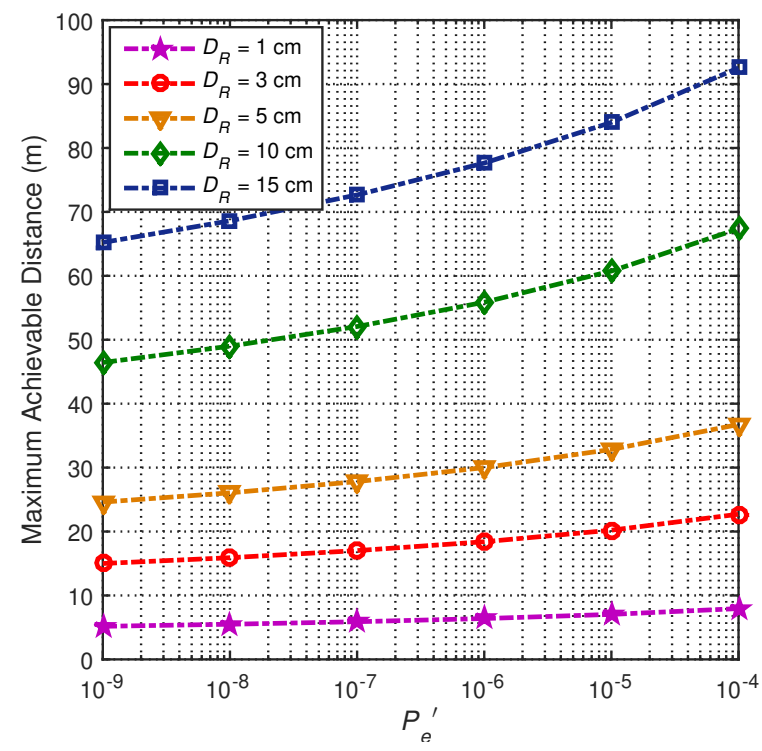

Fig. 7: Maximum achievable distance assuming different receiver aperture diameters

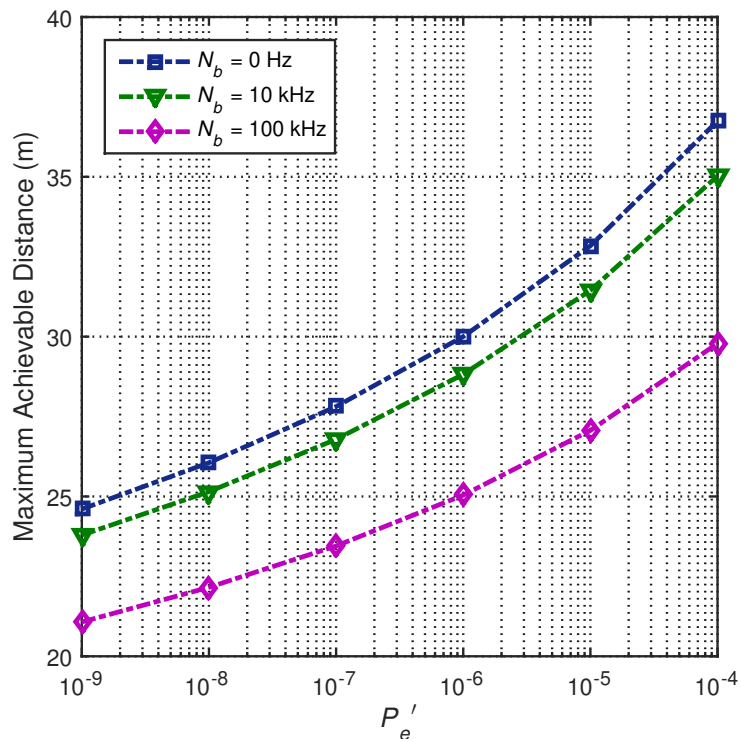

Fig. 8: Maximum achievable distance assuming different background light noise ratios

[7] M. Uysal, Z. Ghassemlooy, A. Bekkali, A. Kadri, and H. Menouar, "Visible light communication for vehicular networking: Performance study of a V2V system using a measured headlamp beam pattern model", IEEE Veh. Technol. Mag., vol. 10, no. 4, pp. 45-53, Dec. 2015.

[8] A. M. Cailean, and M. Dimian, "Impact of IEEE 802.15.7 standard on visible light communications usage in automotive applications", IEEE Commun. Mag., vol. 55, no. 4, pp. 169-175, 2017.

[9] H. Zhang, A. Yang, L. Feng, and P. Guo, "Gb/s real-time visible light communication system based on white LEDs using T-Bridge cascaded pre-equalization circuit", IEEE Photon. J., vol. 10, no. 2, pp. 1-7, April 2018.

[10] M. Ijaz, D. Tsonev, J. J. McKendry, E. Xie, S. Rajbhandari, H. Chun, G. Faulkner, E. Gu, M. D. Dawson, D. O'Brien, and H. Haas, "Experimental proof-of-concept of optical spatial modulation OFDM using micro LEDs", in 2015 IEEE International Conference on Communication Workshop (ICCW), pp. 1338-1343, 2015.

[11] A. M. Cailean, and M. Dimian, "Current challenges for visible light communications usage in vehicle applications: A survey", IEEE Commun. Surveys Tuts., vol. 19, no. 4, pp. 2681-2703, Fourthquarter 2017.

[12] F. Miramirkhani, and M. Uysal, "Channel modeling and characterization for visible light communications", IEEE Photon. J., vol. 7, no. 6, pp: 1-16, 2015.

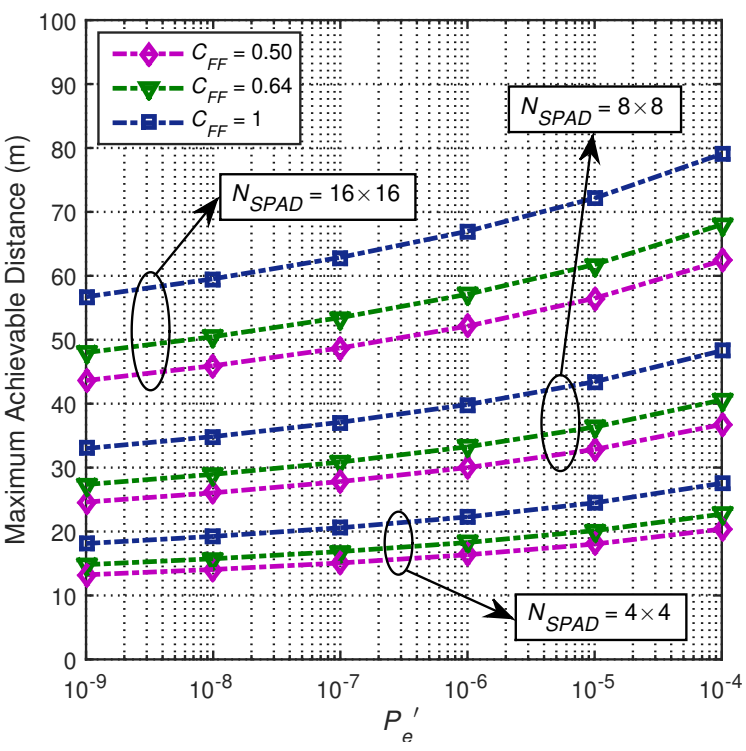

Fig. 9: Maximum achievable distance for different SPAD array parameters (i.e., fill factor and size of the array)

[13] M. Uysal, F. Miramirkhani, O. Narmanlioglu, T. Baykas, and E. Panayirci, "IEEE 802.15.7r1 reference channel models for visible light communications", IEEE Commun. Mag., vol. 55, no. 1, pp: 212-217, 2017.

[14] M. Akanegawa, Y. Tanaka, and M. Nakagawa, "Basic study on traffic information system using LED traffic lights", IEEE Trans. Intell. Transp. Syst., vol. 2, no. 4, pp. 197-203, 2001.

[15] N. Kumar, D. Terra, N. Lourenco, L. N. Alves, and R. L. Aguiar, "Visible light communication for intelligent transportation in road safety applications", in Proc. IEEE Int. Conf. Wireless Communications and Mobile Computing, pp. 1513-1518, 2011.

[16] W. Viriyasitavat, et al., "Short paper: Channel model for visible light communications using off-the-shelf scooter taillight", in IEEE Vehicular Networking Conference (VNC), pp. 170-173, 2013.

[17] S. J. Lee, J. K. Kwon, S. Y. Jung, and Y. H. Kwon, "Evaluation of visible light communication channel delay profiles for automotive applications", EURASIP Journal on Wireless Communications and Networking, 2012:370, pp. 1-8, 2012.

[18] P. Luo, Z. Ghassemlooy, H. L. Minh, E. Bentley, A. Burton, and X Tang, "Fundamental analysis of a car to car visible light communication system", in 9th Int. Symp. Communication Systems, Networks \& Digital Signal Processing (CSNDSP), pp. 1011-1016, 2014.

[19] P. Luo, Z. Ghassemlooy, H. Le Minh, E. Bentley, A. Burton, and X. Tang, "Performance analysis of a car-to-car visible light communication system", Appl. Opt., vol. 54, no. 7, pp. 1696-1706, 2015.

[20] A. Memedi, H. M. Tsai, and F. Dressler, "Impact of realistic light radiation pattern on vehicular visible light communication", in Proc. IEEE Global Telecommunications Conference (GLOBECOM 2017), pp. 1-6, Dec. 2017.

[21] D. Schulz, V. Jungnickel, S. Das, J. Hohmann, J. Hilt, P. Hellwig, A. Paraskevopoulos, and R. Freund, "Long-term outdoor measurements using a rate-adaptive hybrid optical wireless/60 GHz link over $100 \mathrm{~m}$ ", in IEEE 19th Int. Conf. Transparent Optical Networks (ICTON), pp. 1-4, 2017.

[22] Y. H. Kim, W. A. Cahyadi, and Y. H. Chung, "Experimental demonstration of LED-based vehicle to vehicle communication under atmospheric turbulence", in IEEE Int. Conf. Information and Communication Technology Convergence (ICTC), pp. 1143-1145, 2015.

[23] Y. H. Kim, W. A. Cahyadi, and Y. H. Chung, "Experimental demonstration of VLC-based vehicle-to-vehicle communications under fog conditions", IEEE Photon. J., vol. 7, no. 6, pp. 1-9, 2015.

[24] M. Elamassie, M. Karbalayghareh, F. Miramirkhani, R. C. Kizilirmak, and M. Uysal, "Effect of fog and rain on the performance of vehicular visible light communications", in IEEE 87th Vehicular Technology Conference (VTC2018-Spring), Porto, Portugal, 2018.

[25] J. Poliak, P. Pezzei, E. Leitgeb, and O. Wilfert, "Link budget for highspeed short-distance wireless optical link", in 8th IEEE International Symposium on Communication Systems, Networks \& Digital Signal Processing (CSNDSP), pp. 1-6, July 2012. 
[26] M. Uysal, C. Capsoni, Z. Ghassemlooy, A. Boucouvalas, and E. Udvary, Optical wireless communications: an emerging technology, Springer, 2016.

[27] M. Elamassie, F. Miramirkhani, and M. Uysal, "Performance characterization of underwater visible light communication", IEEE Trans. Commun., vol. 67, no. 1, pp. 543-552, 2019.

[28] F. Miramirkhani, O. Narmanlioglu, M. Uysal, and E. Panayirci, "A mobile channel model for VLC and application to adaptive system design", IEEE Commun. Lett., vol. 21, no. 5, pp. 1035-1038, 2017.

[29] Agreement Addendum 111: Regulation No. 112 Revision 3-unece, 2013.

[30] R. E. Stark, "Road surface's reflectance influences lighting design", Light. Design Appl., 1986.

[31] I. I. Kim, B. McArthur, and E. Korevaar, "Comparison of laser beam propagation at $785 \mathrm{~nm}$ and $1550 \mathrm{~nm}$ in fog and haze for optical wireless communications", Proc. SPIE, vol. 4214, pp. 26-37, 2001.

[32] K. Lee, H. Park, and J. R. Barry, "Indoor channel characteristics for visible light communications", IEEE Commun. Lett., vol. 15, no. 2, pp: 217-219, 2011.

[33] P. Dangeti, Statistics for machine learning, Packt Publishing, 2017.

[34] S. Tang, Y. Dong, and X. Zhang, "Impulse response modeling for underwater wireless optical communication links", IEEE Trans. Commun., vol. 62 , no. 1 , pp. 226-234, 2014.

[35] Y. Li, M. Safari, R. Henderson, and H. Haas, "Nonlinear distortion in SPAD-based optical OFDM systems", in IEEE Globecom Workshops (GC Wkshps), San Diego, CA, pp. 1-6, 2015,

[36] Y. Li, M. Safari, R. Henderson, and H. Haas, "Optical OFDM with single-photon avalanche diode", IEEE Photon. Technol. Lett., vol. 27, no. 9, pp. 943-946, 2015.

[37] C. Wang, H. Y. Yu, and Y. J. Zhu, "A long distance underwater visible light communication system with single photon avalanche diode", IEEE Photon. J., vol. 8, no. 5, pp. 1-11, Oct. 2016.

[38] T. Shafique, et al., "Performance analysis of single-photon avalanche diode underwater VLC system using ARQ", IEEE Photon. J., vol. 9, no. 5, pp. 1-11, Oct. 2017.

[39] C. Wang, H. Y. Yu, Y. J. Zhu, and T. Wang, "Blind detection for SPADbased underwater VLC system under $\mathrm{P}-\mathrm{G}$ mixed noise model", IEEE Commun. Lett., , vol. 21, no. 12, pp. 2602-2605, Dec. 2017.

[40] Y. Li, S. Videv, M. Abdallah, K. Qaraqe, M. Uysal, and H. Haas, "Single photon avalanche diode (SPAD) VLC system and application to downhole monitoring", in IEEE Global Communications Conference, Austin, TX, pp. 2108-2113, 2014

[41] M. Karbalayghareh, F. Miramirkhani, M. Safari and M. Uysal, "Vehicular visible light communications with SPAD receivers", in IEEE Wireless Communications and Networking Conference (WCNC), Marrakesh, Morocco, pp. 1-5, 2019.

[42] E. Sarbazi, M. Safari, and H. Haas, "Statistical modeling of singlephoton avalanche diode receivers for optical wireless communications", IEEE Trans. Commun., vol. 66, no. 9, pp. 4043-4058, 2018.

[43] E. Sarbazi, M. Safari, and H. Haas, "Photon detection characteristics and error performance of SPAD array optical receivers", in 4th International Workshop on Optical Wireless Communications (IWOW), pp. 132-136, 2015.

[44] J. J. Shynk, Mathematical foundations for linear circuits and systems in engineering, John Wiley \& Sons, 2016.

[45] E. Fisher, I. Underwood, and R. Henderson, "A reconfigurable singlephoton-counting integrating receiver for optical communications", IEEE J. Solid-State Circuits, vol. 48, no. 7, pp. 1638-1650, 2013.

[46] https://ec.europa.eu/transport/road_safety/specialist/knowledge/speed/ speed_limits/current_speed_limit_policies_en, 2018.

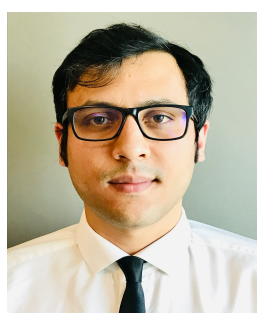

Mehdi Karbalayghareh received the B.Sc. degree in Electrical and Electronics Engineering from Iran University of Science and Technology (IUST), Tehran, Iran, in 2015. He joined Communication Theory and Technologies (CT\&T) Research Group as a research assistant working toward his M.Sc. under the supervision of Prof. Murat Uysal at Ozyegin University, Istanbul, Turkey, in 2016. He received his M.Sc. degree in Electrical and Electronics Engineering in August 2019. He is currently pursuing his Ph.D. degree at The University of Texas at Dallas, Richardson, TX, USA. His current research interests include information theory, cooperative communications, optical wireless communications, multipleinput multiple-output systems, resource allocation, and energy harvesting.

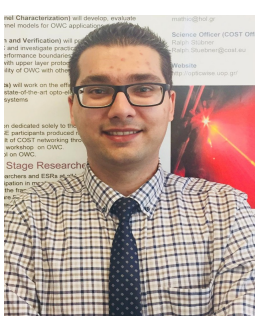

Farshad Miramirkhani received the B.Sc. and M.Sc. degrees (Hons.) in Electrical and Electronics, and Communication Engineering from University of Isfahan, Isfahan, Iran, in 2011 and 2014, respectively. He joined Communication Theory and Technologies (CT\&T) Research Group as a research assistant working toward his doctorate under supervision of Prof. Murat Uysal at Ozyegin University, Istanbul, Turkey, in 2014. He received his Ph.D. degree (Hons.) in Electrical and Electronics Engineering in June 2018. Currently, he is an assistant professor at Isik University, Department of Electrical and Electronics Engineering. He serves as Telecommunications Advisory Board Member of Cambridge Scholars Publishing, Academic Editor of IntechOpen and Editorial Board Member of Optical Communications-Clausius Scientific Press. He has also contributed to the standardization works of IEEE 802.15.13 (Short Range Optical Wireless Communications) and IEEE 802.11bb (Light Communications for Wireless Local Area Networking). The LiFi channels developed by Prof. Murat Uysal and Dr. Miramirkhani were selected as the LiFi Reference Channel Models by the IEEE 802.15.13 and IEEE 802.11bb Task Groups. His current research interests include optical wireless communications, indoor VLC, underwater VLC, vehicular VLC and channel modeling.

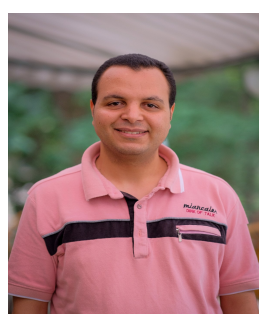

Hossien Eldeeb received the B.Sc. degrees in Electronics and Electrical Communication engineering from Menoufia University, Menouf, Egypt, in 2008, and the M.Sc. degrees in Electrical and Electronics Engineering from Cairo University, Cairo, Egypt, in 2017. He is currently pursuing the Ph.D. degree with the Communication Theory and Technologies (CT\&T) Research Group, Ozyegin University, Istanbul, Turkey, under the supervision of Prof. M. Uysal. He joined the CT\&T Research Group as a Research Assistant in 2018. He has served as an Instrumentation \& Control Engineer with Cairo-West and Giza-North Electrical Power Stations, Giza, Egypt. His current research interests include optical wireless communications, channel modeling for indoor and outdoor VLC, and diversity techniques.

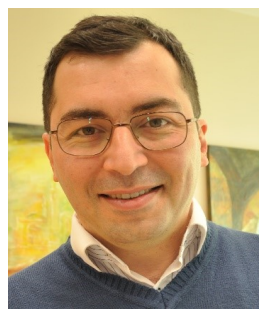

Refik Caglar Kizilirmak (M'12) received the B.Sc. and M.Sc. degrees in Electrical and Electronics Engineering from Bilkent University, Ankara, Turkey, in 2004 and 2006, respectively, and the Ph.D. degree from Keio University, Yokohama, Japan, in 2010. He worked for the Communications and Spectrum Management Research Center, Turkey, on several telecommunication and defense industry projects. $\mathrm{He}$ contributed to the technical requirements document of 802.15.7r1 standardization, which will enable VLC. He has three patent applications in the field of wireless communications to US and Japan patent offices. Currently, he is with the Department of Electrical and Electronics Engineering, Nazarbayev University, Astana, Kazakhstan. He is the recipient of IEEE VTS Japan 2008 Young Researcher's and IEICE WBS Best Paper Awards.

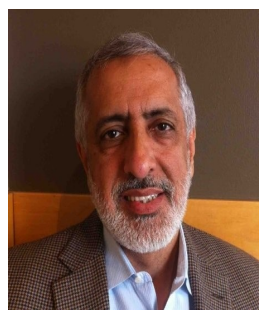

Sadiq M. Sait (SM'02) was born in Bengaluru. He received the bachelor's degree in electronics engineering from Bangalore University in 1981, and the master's and Ph.D. degrees in electrical engineering from the King Fahd University of Petroleum \& Minerals (KFUPM) in 1983 and 1987, respectively. $\mathrm{He}$ is currently a Professor of Computer Engineering and the Director of the Center for Communications and IT Research, Research Institute, KFUPM. He has authored over 200 research papers, contributed chapters to technical books, granted several US patents, and lectured in over 25 countries. He is also the Principle Author of two books. He received the Best Electronic Engineer Award from the Indian Institute of Electrical Engineers, Bengaluru, in 1981. 


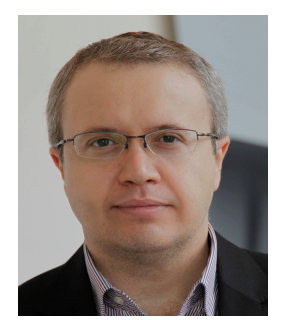

Murat Uysal received the B.Sc. and M.Sc. degrees in electronics and communication engineering from Istanbul Technical University, Istanbul, Turkey, in 1995 and 1998, respectively, and the Ph.D. degree in electrical engineering from Texas A\&M University, College Station, TX, USA, in 2001. He is currently a Full Professor and the Chair of the Department of Electrical and Electronics Engineering with Ozyegin University, Istanbul. He also serves as the Founding Director of the Center of Excellence in Optical Wireless Communication Technologies. Prior to joining Ozyegin University, he was a tenured Associate Professor with the University of Waterloo, Canada, where he still holds an adjunct faculty position. He has authored some 290 journal and conference papers in his research topics and received more than 7500 citations. His research interests are in the broad areas of communication theory and signal processing with a particular emphasis on the physical-layer aspects of wireless communication systems in radio and optical frequency bands. His distinctions include the Marsland Faculty Fellowship in 2004, the NSERC Discovery Accelerator Supplement Award in 2008, the University of Waterloo Engineering Research Excellence Award in 2010, the Turkish Academy of Sciences Distinguished Young Scientist Award in 2011, and the Ozyegin University Best Researcher Award in 2014. He currently serves on the editorial board of the IEEE TRANSACTIONS ON WIRELESS COMMUNICATIONS. In the past, he was an Editor of the IEEE TRANSACTIONS ON COMMUNICATIONS, the IEEE TRANSACTIONS ON VEHICULAR TECHNOLOGY, the IEEE COMMUNICATIONS LETTERS, Wireless Communications and Mobile Computing Journal, and the Transactions on Emerging Telecommunications Technologies, and a Guest Editor of the IEEE JOURNAL ON SELECTED AREAS IN COMMUNICATIONS Special Issues on Optical Wireless Communication (2009 and 2015). He was involved in the organization of several IEEE conferences at various levels. He served as the Chair of the Communication Theory Symposium of IEEE ICC 2007, the Chair of the Communications and Networking Symposium of IEEE CCECE 2008, the Chair of the Communication and Information Theory Symposium of IWCMC 2011, a TPC Co-Chair of the IEEE WCNC 2014, and the General Chair of the IEEE IWOW 2015. Over the years, he has served on the technical program committee of more than 100 international conferences and workshops in the communications area. 